\title{
MRI evaluation of fusion mass incorporation after anterior cervical bony fusions: preliminary findings
}

\author{
T J Albert MD, ${ }^{1}$ D Lamb MD,${ }^{1}$ M R Piazza MD,${ }^{3}$ A E Flanders MD,${ }^{2}$ R A Balderston \\ $\mathrm{MD},{ }^{4} \mathrm{~J}$ M Cotler $\mathrm{MD}^{1}$
}

${ }^{1}$ Department of Orthopaedic Surgery, ${ }^{2}$ Department of Radiology, Thomas Jefferson University 1015 Walnut Street, Philadelphia, PA 19107, USA; ${ }^{3}$ Orthopaedic Surgery of Clearwater, Clearwater, FL 34616, USA; ${ }^{4}$ Department of Orthopaedics, Pennsylvania Hospital, 800 Spruce Street, Philadelphia, PA 19107, USA.

While Modic ${ }^{3}$ has described certain MRI changes in bone grafts in patients after anterior cervical discectomy and fusion (ACF), no study has evaluated specific MRI signal changes in the fusion mass prospectively and longitudinally. The goal of the present study was to prospectively evaluate MRI changes in ACF grafts in a longitudinal fashion.

Twenty-seven MRIs were included in the evaluation of seven postoperative patients. MRIs were obtained in a 1.5 tesla GE imager immediately postoperatively, and at 1, 3, and 6 months in most patients. All patients received at least three sequential MRIs. Two patients had greater than one level fused. T1 and T2 images were evaluated in all patients at each available interval. GRASS images were not found to be helpful secondary to a prohibitive amount of noise.

For single level fusion masses, T1 images showed a very intense homogeneous graft signal in the immediate postoperative period which decreased slightly at 1 month. At 3 months the signal intensity of the graft was similar to the 1 month image, but was slightly less homogeneous. At 6 months, the T1 signal had greatly decreased in much of the graft. Immediate postoperative specimens showed heterogeneously high signal on T2 images which increased at 1 month in the whole graft, and then seemed to increase at the endplates while decreasing in the graft at 3 months. At 6 months the graft was becoming difficult to delineate on T2 images in many specimens. The two and three level fusions had a more heterogeneous image progression without clear evidence of solid fusion at 6 months. One patient with a C3-4, C4-5, C5-6 fusion still showed heterogeneous intensity of both $\mathrm{T} 1$ and $\mathrm{T} 2$ signals at all levels 2 years postoperatively while remaining asymptomatic.

This study documents specific progression of time-related signal changes in bone grafts after ACF. The identification of signals predictive of graft failure will require more prefailure MRIs of grafts which ultimately go on to pseudarthrosis. Multiple level fusions, known to have a lower fusion rate, demonstrate signal patterns dissimilar to single level fashions.

Keywords: MRI; cervical bony fusion, pseudarthrosis; spine.

\section{Introduction}

Surface coil magnetic resonance imaging (MRI) has become an indispensable tool for orthopaedic surgeons. The indications for use of MRI include the work-up of radiculo-

Correspondence: c/o Rothman Institute, 800 Spruce Street, Philadelphia, PA 19107, USA. pathy, myelopathy, neoplasms and trauma. ${ }^{1-3}$ Given the unique ability to provide high contrast between adjacent soft tissues as well as specific signal changes with regard to differential contents of fat and water in the tissues, and advantages regarding plane of section and orientation, MRI may be potentially useful in the evaluation of bone grafts in the postoperative patient. 
The viability of acetabular bone grafts in total hip arthroplasty has already been studied utilizing MRI. ${ }^{4}$

Ross and colleagues attempted to evaluate changes in the postoperative cervical spine using MRI in a retrospective fashion. ${ }^{5}$ They evaluated 73 postoperative cervical spine patients and correlated MRIs postoperatively with their initial operative reports. The investigators found a spectrum of graft and vertebral body signal changes up to 2 years following anterior cervical fusion. They also found that the fusion mass was incorporated at greater than 2 years postoperatively. They described a number of postoperative complications that were discernible using MRI. Only four patients in the Ross study were imaged in a longitudinal fasion and only two of these patients had anterior cervical fusions. They were imaged preoperatively, at 10 days postoperatively, and then at 8 months postoperatively.

The goal of the present study was to prospectively evaluate the use of magnetic resonance imaging in the postoperative anterior cervical fusion (ACF) patient in a longitudinal fashion. We would ultimately like to define time related signal changes in the fusion mass and to be able to use these time related signal changes as predictors of ultimate success or failure of the anterior cervical fusion.

\section{Methods}

MRI scans were obtained in anterior cervical fusion patients in the immediate postoperative period, at 1 month, 3 months, and 6 months after surgery. A GE Cigna 1.5 tesla magnet was used for evaluation. All MRIs were reviewed by a neuroradiologist and an orthopaedist. The data were tabulated and compared with multiple additional nonlongitudinal studies.

The images were evaluated specifically for signal changes on $\mathrm{T} 1$ and $\mathrm{T} 2$ weighted scans in the graft itself, at the edges of the graft, in the bordering vertebral bodies, and in the remainder of the spine. Results were tabulated according to level of ACF and time postoperatiely at which the MRI was obtained.
Office charts and xrays were reviewed to assess the clinical postoperative course of each patient included in the study. The time at which fusion was felt to be solid by plain xray or tomography was recorded along with the time at which the patient was taken out of his/her Philadelphia collar.

\section{Results}

The results include the analysis of $26 \mathrm{MRIs}$ from seven subjects. All of these MRIs were taken in a longitudinal fashion and at least three MRIs were obtained for each subject. The T1 and T2 spin echo images were used for analysis. Gradient recalled acquisition at steady state or GRASS imaging was not found to be useful secondary to a great deal of artifact and was, therefore, excluded from the study. All patients underwent anterior cervical fusion (ACF) by the SmithRobinson technique. ${ }^{6}$ Two patients included in the study had multiple level fusions. One patient had multiple grafts placed at surgery while the second patient had a C5-6 fusion 7 years prior to the index procedure at which time he had a $\mathrm{C} 4-5$ and $\mathrm{C} 6-7$ fusion. The remaining five patients had single level ACFs. The signal changes seen in the single level fusions were generally constant.

No patient in the study was felt to have a pseudarthrosis by plain xray or tomographic examination. No patient remained in a Philadelphia collar for longer than 12 weeks.

The typical immediate postoperative MRI (Fig 1) showed a strong hyperintense signal on the T1 image and an isointense or speckled image on $\mathrm{T} 2$ when comparing the graft to the adjacent vertebral bodies. At approximately 1 month postoperatively the T1 signal showed a decreasing but still hyperintense signal while the T2 image showed an increasing or isointense signal (Fig 2).

At 3 months postoperatively the graft on T1 imaging was isointense with the graft signal seen at 1 month (Fig 3). On T2 imaging, the fusion mass was noted to have a decreasing signal while the adjacent vertebral endplates showed an increased signal during this period. At approximately 6 months postoperatively the signal on T1 


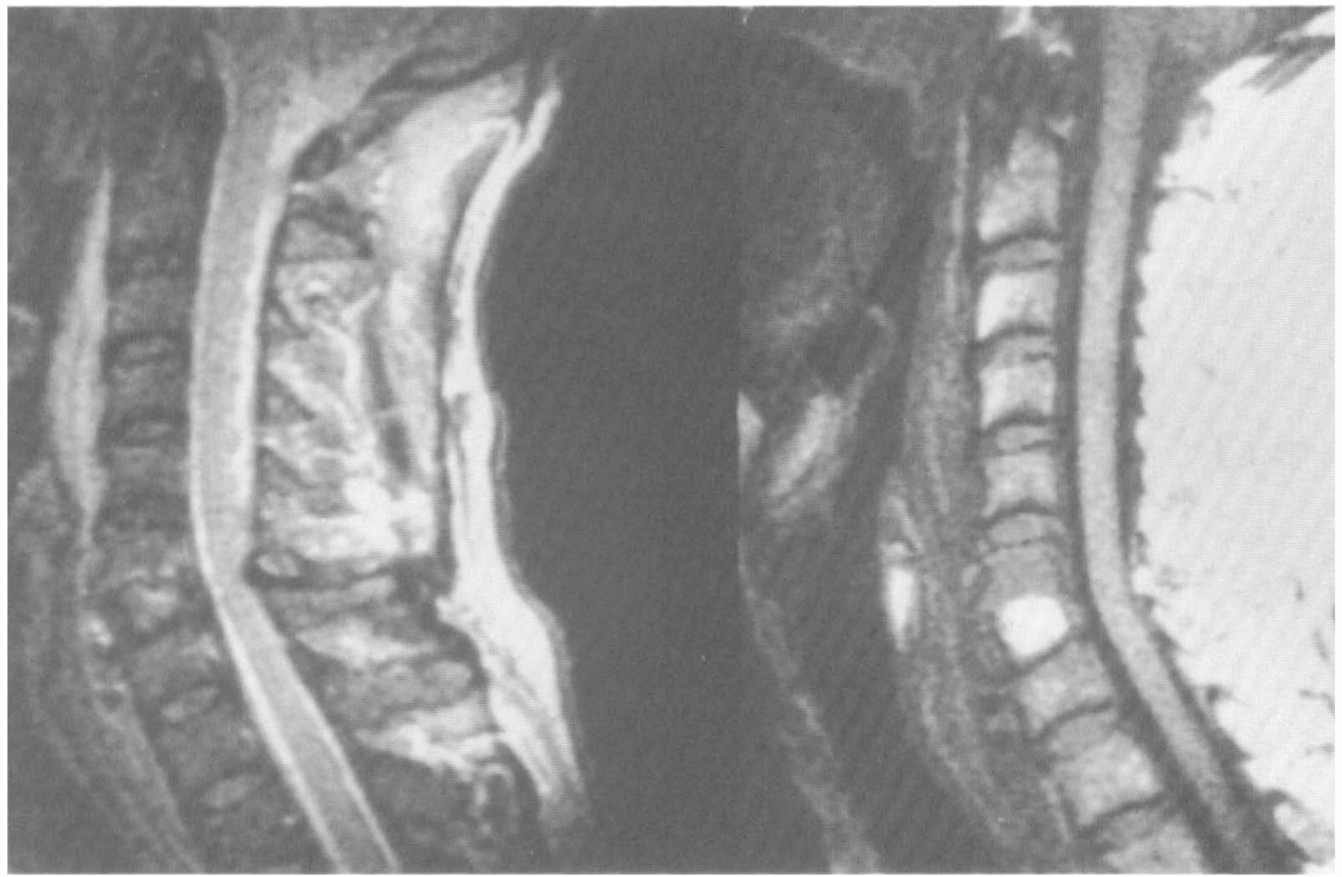

Figure 1 Case 1. Immediate postoperative MRI: T2 weighted image on the left, T1 on the right. The graft is speckled on $\mathrm{T} 2$ and hyperintense on T1 images.



Figure 2 Case 2. One month postoperatively. An increasing T2 signal in the graft on the left and a decreasing but hyperintense T1 signal on the right. 


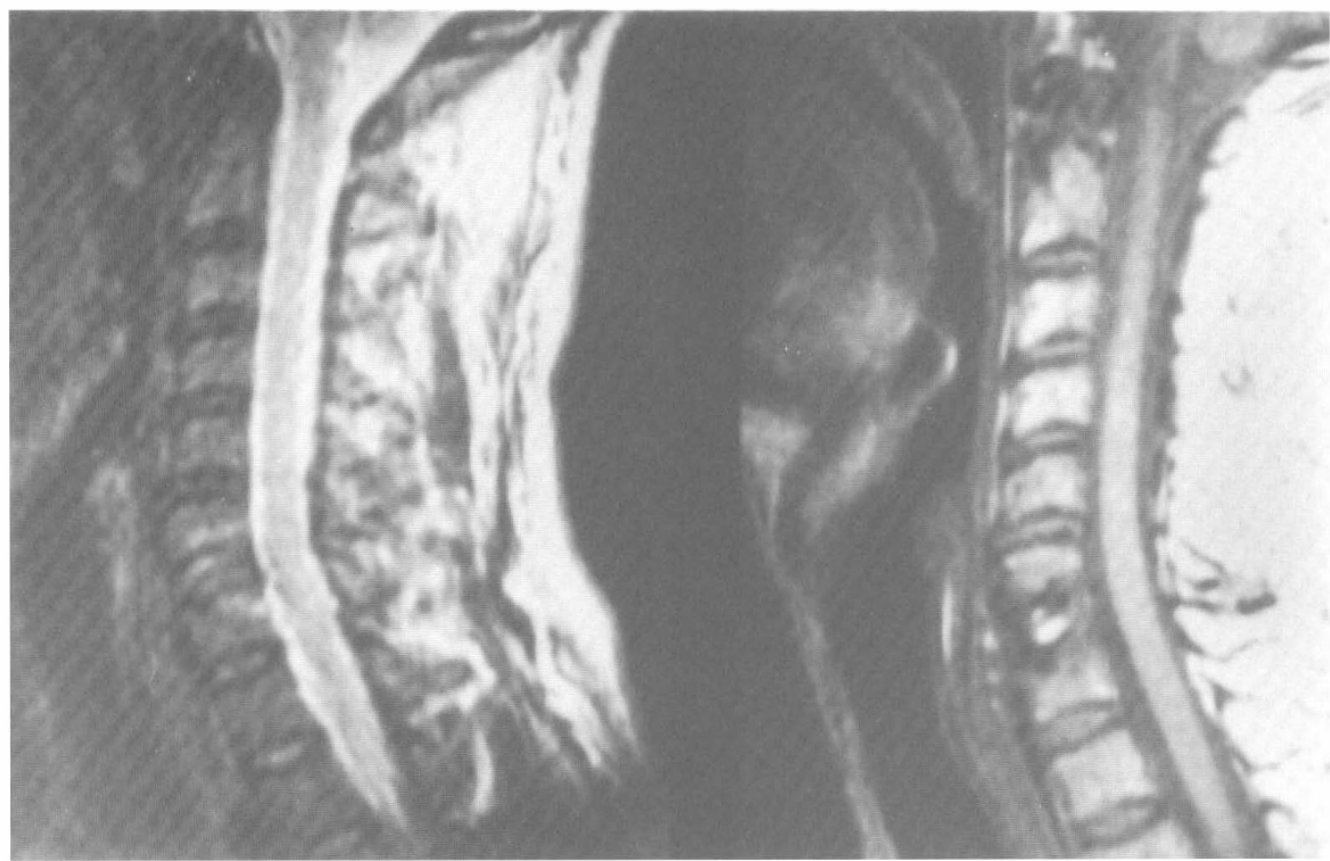

Figure 3 Case 1 . Three months postoperatively. The T1 image is isointense in the graft with the 1 month image. The T2 image (left) shows hyperintensity at the vertebral endplates on either side of the graft with the graft signal decreasing.

imaging emanating from the graft was the lowest seen and the T2 signal of the graft became isointense with the vertebral bodies (Fig 4). The graft at this time was actually difficult to see.

The two patients in the study with multiple fusion levels provided an interesting contrast to the single level fusion patients. There was a significant increase in heterogeneity in the signal changes between levels on both $\mathrm{T} 1$ and $\mathrm{T} 2$ images in the multiple level fusions. In a patient with a $\mathrm{C} 3-4$, C4-5, C5-6 fusion, the immediate postoperative MRI showed decreasing T1 signal intensity at the C3-4 graft with hyperintensity of the C4-5 and C5-6 grafts. The heterogeneity of signal in this patient increased to that seen in Figure 5 at 3 months with varying graft and body signals and collapse of C6. At 2 years postoperatively, evidence of consolidation without full fusion is apparent with ongoing heterogeneity of signal on T1 and T2 images (Fig 6).

Another patient with a C4-5, C5-6,
C6-7 ACF showed a heterogeneous signal pattern between the upper level graft and the two lower level grafts. At 13 months postoperatively on T1 imaging, this patient's upper graft had an increasing signal intensity while the lower two levels show very hypointense signal on T1. On T2 imaging the upper level $(\mathrm{C} 4-5)$ was isointense with the other vertebral bodies, while the C5-6 and C6-7 level showed a decreased signal intensity compared to the vertebral bodies.

\section{Discussion}

This preliminary study has shown specific signal changes on T1 and T2 weighted MRI imaging of anterior cervical fusions at specific time intervals (Table I). The T1 signal shows an initial signal increase after graft implantation with slowly decreasing signal intensity at up to 6 months postoperatively. This decreasing signal may represent the elution of fatty marrow from the initially 


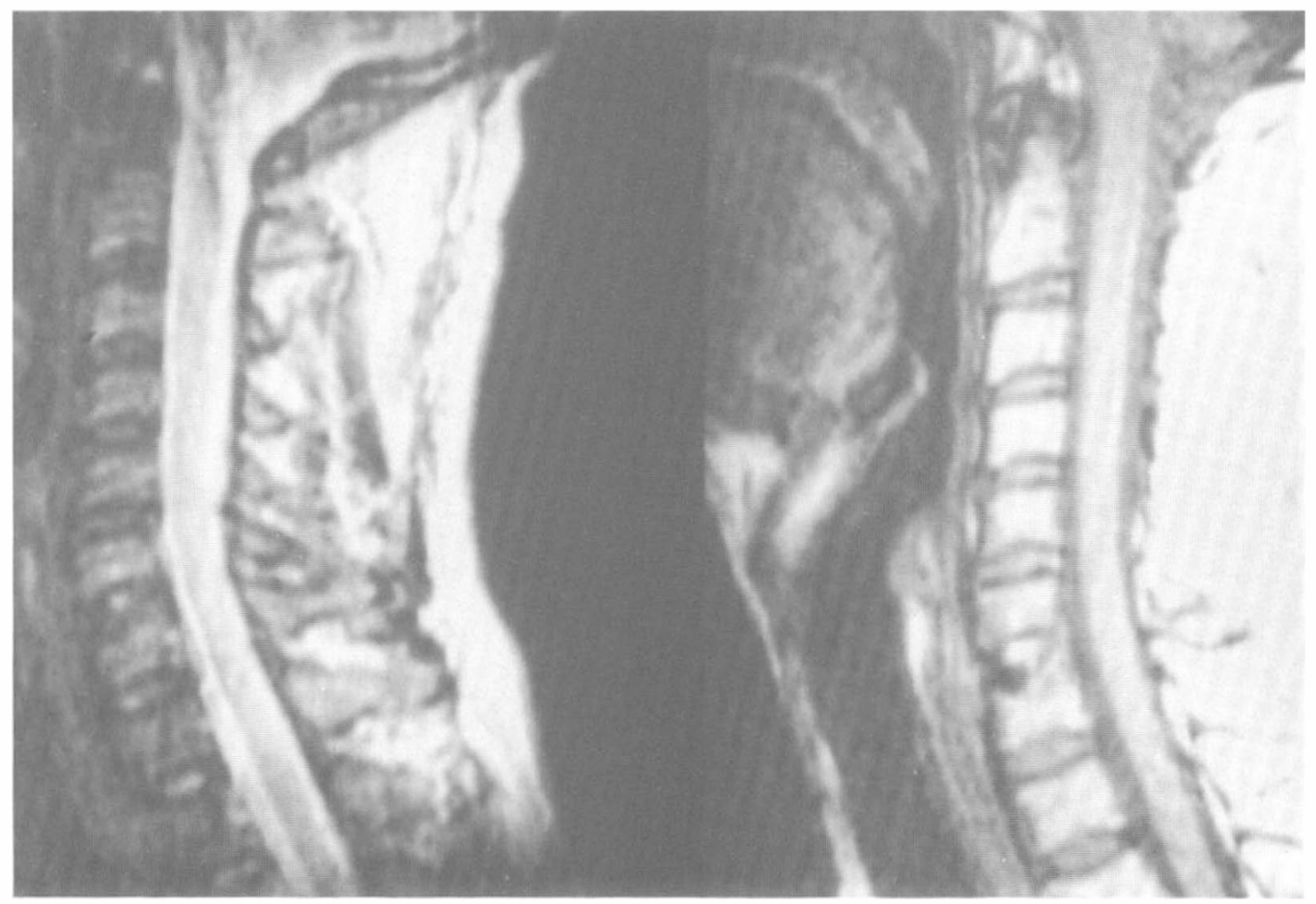

Figure 4 Case 1. Six months postoperatively. T1 (right) shows the lowest graft signal of the preceding images. T2 shows isointense signal with difficulty in delineating the graft.

placed graft as the graft becomes incorporated with a body above and below. The T2 signal shows an initial speckling or isointensity with the other vertebral bodies and this signal increases over time to become essentially isointense with the surrounding bodies at 6 months, making the graft difficult to see. This may also represent the ongoing incorporation of a single level fusion at up to 6 months postoperatively in our small population. The increasing signal intensity at 3 months on the T2 image, especially at the endplates, may represent an aseptic inflammatory process due to ongoing instability versus a manifestation of the normal reparative process of graft incorporation into the adjacent vertebral bodies. We postulate that the signal changes seen in the study relate to the following: (1) the initial status of the vertebral body or graft, be it fatty or aqueous; (2) the amount of trauma to the remaining endplate, vertebral body, or graft; (3) the postoperative stresses placed on the fusion mass; (4) the degree of revascularization at any point in time. Certainly the identification of any one signal as a predictor of future fusion is difficult in the absence of a larger sample size or the inclusion of prefailure MRIs of ultimately failed single level fusions. We hypothesize that changes identified here represent the normal progression of MRI signal changes with the ultimate fusion incorporating successfully.

The variability of signal changes between graft levels in the two patients with multiple level fusions may suggest ongoing instability in the grafts. It has been shown that multilevel fusions have a higher rate of pseudarthrosis. ${ }^{6,7}$ Again longer term follow up MRIs to determine ultimate success or failure of these multilevel fusions will help to identify predictive signal changes which would herald failure of the fusion. Furthermore, radiographic failure of fusion does not necessarily correlate with a poor clinical result.

Ross et al found a spectrum of graft and 


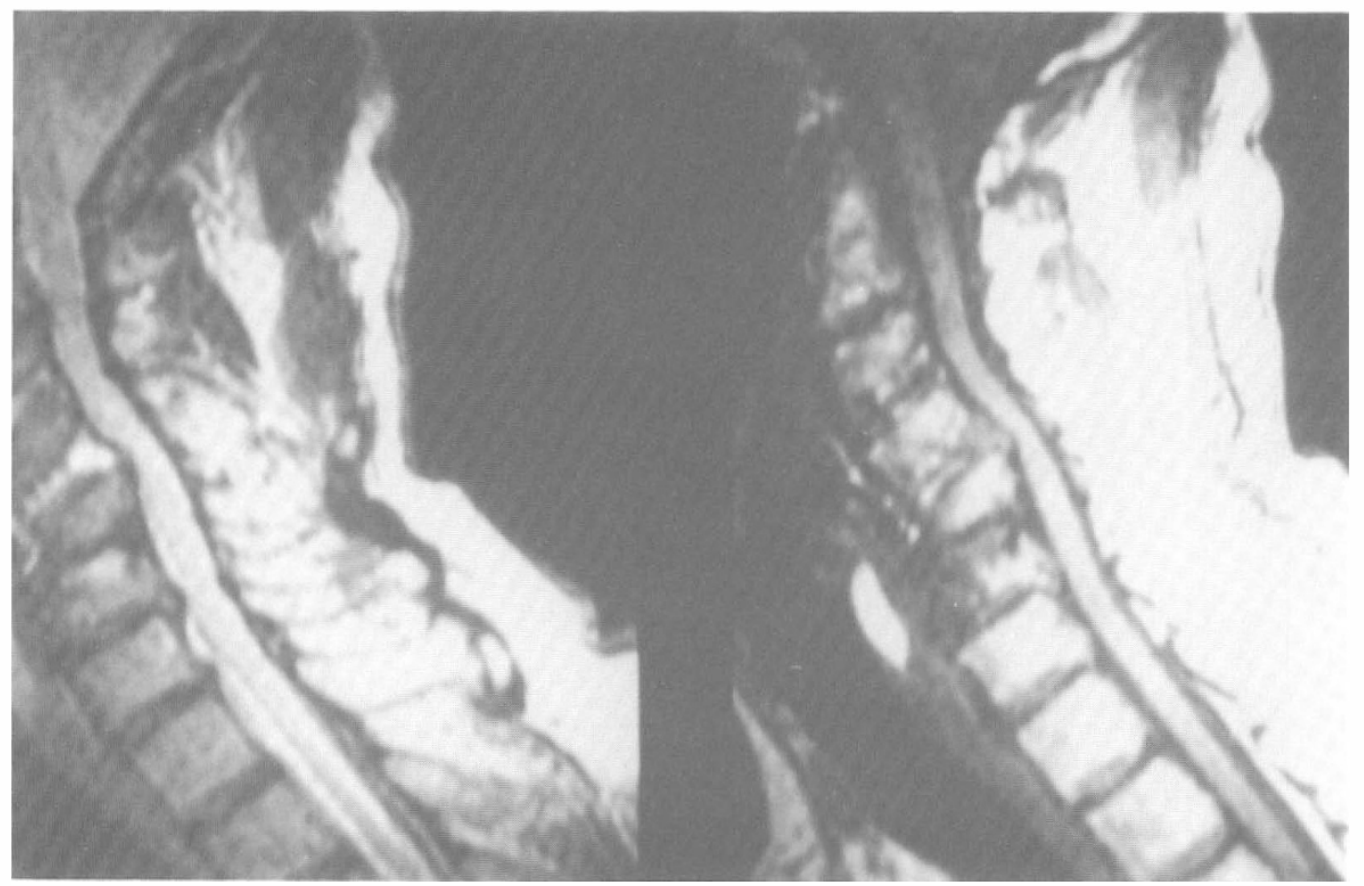

Figure 5 This patient with multiple graft levels (3) shows heterogeneous signal on both $\mathrm{T} 1$ on the right and T2 on the left between graft levels. Also note vertebral collapse and cord impingement. Patient was asymptotic at the time of MRI.

vertebral body changes on T1 and T2 MRI images over time. ${ }^{5}$ The vast majority of their 73 images were nonlongitudinal. However, their results in general corroborated the signal changes found in the present study. They did find that after 2 years postoperatively, all MRI scans showed evidence of solid bony fusion without evidence of graft or disc space visible. None of our prospective longitudinal patients showed the complete loss of disc space or delineation of the graft as is shown in a nonlongitudinal patient at 18 months postoperatively from a C3 through C6 ACF (Fig 7). This type of image seen in all of our patients with single level fusion would confirm that the progression of signal changes seen in these patients represented a natural progression to bony fusion. This probably requires longer term follow up with more longitudinal MRIs.

Lang and associates studied the MRI qualities of lumbar vertebral bodies after fusion. ${ }^{8}$ The majority of their patients had posterolateral lumbar fusions but their aim was to predict stability versus instability of the vertebral bodies. What they identified as stable lumbar vertebrae showed a hyperintensity of a T1 signal and an iso- or hypointense $\mathrm{T} 2$ signal at 1 year postoperatively. Those patients designated with an unstable fusion had hypointensity of the T1 signal and hyperintensity of $\mathrm{T} 2$ signal which they felt was suggestive or inflammation, fibrosis, or hyperemia. Our graft signals on T1 imaging became increasingly less intense while T2 signals became relatively increasingly intense. This finding agrees with Lang's data with regard to stability as the intensity of our signal was compared to the adjacent vertebral bodies suggesting a concomitant relative increase in the T1 signal of the adjacent vertebral bodies with time and a relative decrease in the $\mathrm{T} 2$ signal intensity in the adjacent vertebral bodies for single level fusion patients over time.

In conclusion, longitudinal study of ACFs in the postoperative period is potentially 


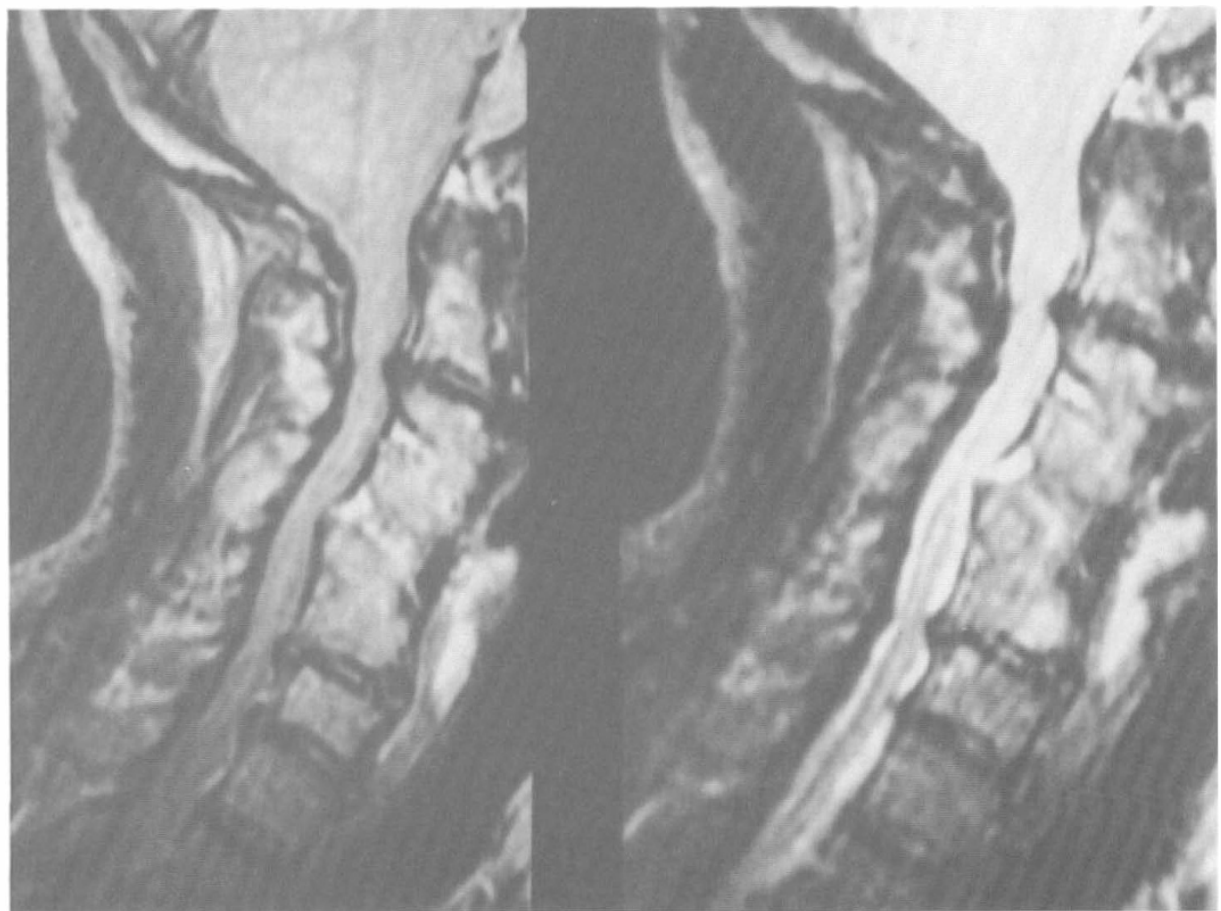

Figure 6 Same patient as in Figure 5 at 2 years postoperatively showing less heterogeneity of signal, canal impingement, and without complete fusion. Image on the left is proton density. T2 on the right.

Table I Summary of graft MRI changes in single level ACFs over time

\begin{tabular}{lllll}
\hline & Immediate & 1 month & 3 months & 6 months \\
\hline T1 & Hyperintense signal & $\begin{array}{l}\text { Decreasing signal still } \\
\text { hyperintense }\end{array}$ & $\begin{array}{l}\text { Same as 1 month } \\
\text { signal }\end{array}$ & $\begin{array}{l}\text { Low signal (still } \\
\text { hyperintense com- } \\
\text { pared to vertebral } \\
\text { bodies) }\end{array}$ \\
T2 & $\begin{array}{l}\text { Isointense/speckled } \\
\text { signal }\end{array}$ & $\begin{array}{l}\text { Increasing or iso- } \\
\text { intense with vertebral } \\
\text { bodies }\end{array}$ & $\begin{array}{l}\text { Graft signal decreas- } \\
\text { ing, endplates signal } \\
\text { increasing }\end{array}$ & $\begin{array}{l}\text { ral bodies } \\
\text { ralth verteb- }\end{array}$ \\
\hline
\end{tabular}

useful in attempting to predict the ultimate success or failure of fusion using MRI analysis. However, the ultimate prediction of failure or success will require more prefailure MRIs of failed ACFs as well as further follow up of patients going on to solid arthrodesis with reliable and repeatable signal changes. The heterogeneity of patients with multiple grafts may suggest ongoing instability and the increased risks of pseudarthrosis with increasing number of graft levels.

We do not at this time recommend the routine use of interval MRI in the postoperative routine ACF patient. Rather, this tool may potentially be useful in those patients with suspected problems. The predictable signal changes preceding solid arthrodesis should not be confused with pseudarthrosis or infection. 


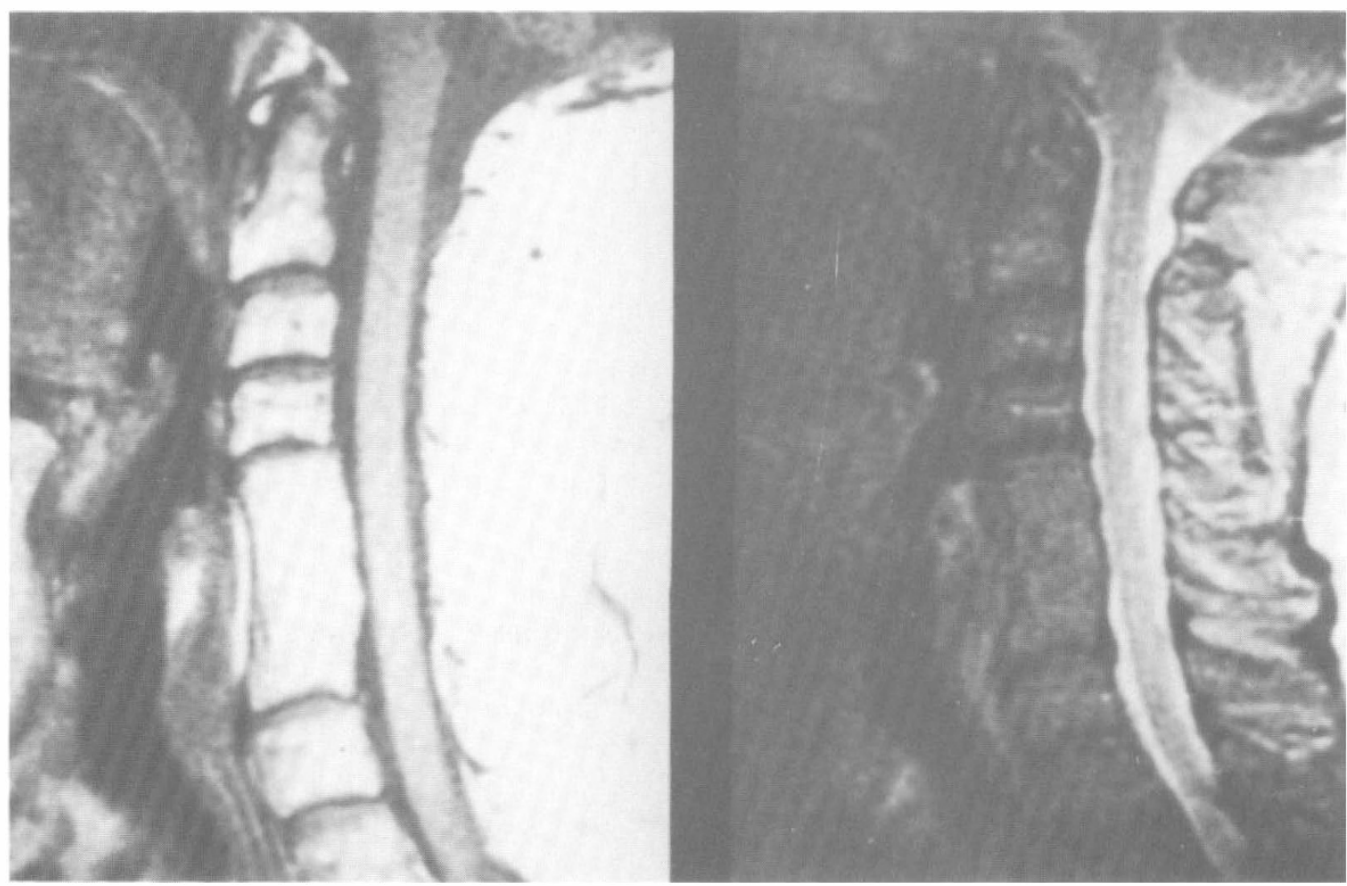

Figure 7 A single MRI of a patient not reviewed in this study demonstrates a solid arthrodesis with inability to identify the disc spaces and homogeneity of signal acoss the multilevel fusion.

\section{References}

1 Ehman RL, Berquist TH, McLeod RA (1988) MR imaging of the musculoskeletal system: A five year appraisal. Radiology 166: 313-320.

2 Haughton VM (1988) MR imaging of the spine. Radiology 166: 297-301.

3 Modic MT, Steinberg PM, Ross JS, Masaryk TJ, Carter JR (1988) Degenerative disc disease: Assessment of changes in vertebral body marrow with MR imaging. Radiology 166: 193-199.

4 Pedrosa P, Wenda K, Higer HP, Ritter G (1989) Bone grafting in total hip replacement: Preliminary results with MRI. Eur J Radiol 9: 12-16.

5 Ross JS, Masaryk TJ, Modic MT (1987) Postoperative cervical spine: MR assessment. J Comput Assist Tomogr 11(6): 955-962.

6 Robinson RA, Walker AE, Ferlic DC, Wiecking DK (1962) The results of an anterior interbody fusion of the cervical spine. J Bone Joint Surg 44(A): 1569 .

7 White AA, III, Southwick WO, DePonte RJ, Gainor JW, Hardy R (1973) Relief of pain by anterior cervical spine fusion for spondylosis: A report of 65 cases. J Bone Joint Surg 55(A): 525.

8 Lang P, Chafetz N, Genant HK, Morris JM (1990) Lumbar spinal fusion: Assessment of functional stability with magnetic resonance imaging. Spine 15(5): 581-587. 